\title{
Development of a rapid SNP-typing assay to differentiate Bifidobacterium animalis ssp. lactis strains used in probiotic-supplemented dairy products
}

\author{
Sara Lomonaco, ${ }^{*} \dagger$ Emily J. Furumoto, $\dagger$ Joseph R. Loquasto, $†$ Patrizia Morra, ${ }^{*}$ Ausilia Grassi, ${ }^{*}$ \\ and Robert F. Roberts $\dagger^{1}$ \\ *Dipartimento di Scienze Veterinarie, Università degli Studi di Torino, 10095 Grugliasco TO, Italy \\ †Department of Food Science, The Pennsylvania State University, University Park 16802
}

\begin{abstract}
Identification at the genus, species, and strain levels is desirable when a probiotic microorganism is added to foods. Strains of Bifidobacterium animalis ssp. lactis (BAL) are commonly used worldwide in dairy products supplemented with probiotic strains. However, strain discrimination is difficult because of the high degree of genome identity $(99.975 \%)$ between different genomes of this subspecies. Typing of monomorphic species can be carried out efficiently by targeting informative single nucleotide polymorphisms (SNP). Findings from a previous study analyzing both reference and commercial strains of BAL identified SNP that could be used to discriminate common strains into 8 groups. This paper describes development of a minisequencing assay based on the primer extension reaction (PER) targeting multiple SNP that can allow strain differentiation of BAL. Based on previous data, 6 informative SNP were selected for further testing, and a multiplex preliminary PCR was optimized to amplify the DNA regions containing the selected SNP. Extension primers (EP) annealing immediately adjacent to the selected SNP were developed and tested in simplex and multiplex PER to evaluate their performance. Twenty-five strains belonging to 9 distinct genomic clusters of $B$. animalis ssp. lactis were selected and analyzed using the developed minisequencing assay, simultaneously targeting the 6 selected SNP. Fragment analysis was subsequently carried out in duplicate and demonstrated that the assay yielded 8 specific profiles separating the most commonly used commercial strains. This novel multiplex PER approach provides a simple, rapid, flexible SNP-based subtyping method for proper characterization and identification of commercial probiotic strains of BAL from fermented dairy products. To assess the usefulness of this method, DNA was extracted from yogurt manufactured with and without the addition of B. animalis ssp. lactis BB-12. Extracted DNA was then
\end{abstract}

Received June 20, 2014.

Accepted November 18, 2014.

${ }^{1}$ Corresponding author: rfr3@psu.edu subjected to the minisequencing protocol, resulting in a SNP profile matching the profile for the strain BB- 12 . Key words: Bifidobacterium animalis ssp. lactis, probiotic, single nucleotide polymorphism (SNP) minisequencing, molecular subtyping

\section{INTRODUCTION}

Bifidobacterium lactis was originally isolated as a moderately oxygen-tolerant species from a commercial yogurt sample (Meile et al., 1997). Bifidobacterium animalis ssp. lactis, as it was later reclassified (Masco et al., 2004), exhibits certain technological advantages, such as acid and oxygen tolerance, compared with other species within the same genus. These characteristics allow for its successful inclusion in commercial food products (Matsumoto et al., 2004; Simpson et al., 2005; Vernazza et al., 2006). Health-promoting benefits have also been attributed to strains of this subspecies, including reduced gut transit time and immune modulation (Marteau et al., 2002; Veiga et al., 2010; Waller et al., 2011). Because health benefits attributed to probiotic microorganisms are currently considered to be strain-specific (FAO/WHO, 2002), health benefits attributed to a strain via clinical studies may not be extrapolated to other strains of the same species or subspecies. Therefore, it is critical for suppliers of probiotics and manufacturers of probiotic-containing product to be able to verify that the strain of $B$. animalis ssp. lactis claimed is, in fact, the strain actually present in the product. Historically, this has not been a simple task because of the phenotypic and genotypic similarity of different strains. Methods for strain-level differentiation such as pulsed-field gel electrophoresis (PFGE), randomly amplified polymorphic DNA-PCR (RAPDPCR), and multi-locus sequence typing (MLST) based on conserved gene sequences do not have sufficient discriminatory power to properly differentiate strains of this subspecies (Roy and Sirois, 2000; Jian et al., 2001; Ventura and Zink, 2003; Briczinski and Roberts, 2006).

The complete genomes of several strains of B. animalis ssp. lactis have been sequenced, including most of 
the widely used commercial strains (Barrangou et al., 2009; Kim et al., 2009; Garrigues et al., 2010; Sun et al., 2010; Bottacini et al., 2011; Chervaux et al., 2011; Stahl and Barrangou, 2012). Sequencing revealed that the genomes of these strains exhibit remarkable homogeneity despite unique chronological and geographical isolations complicating strain identification and differentiation. Only recently, a unique strain containing novel genetic content was sequenced and described (Loquasto et al., 2013).

By comparing the genome sequences of 2 strains of B. animalis ssp. lactis, Briczinski et al. (2009) were able to develop a method based on SNP-INDEL (insertion/ deletion) to differentiate strains. This SNP-INDEL typing scheme provided a reliable method to differentiate strains among this subspecies. However, to perform the analysis, each of 6 informative loci had to be individually amplified using PCR, electrophoresed, purified, and sequenced, a time consuming and expensive process.

One approach to make the SNP analysis faster is minisequencing based on single nucleotide primer extension reactions (PER). Such an approach has been widely applied in the last few years for rapid differentiation of some human pathogens, including Vibrio species, Listeria species, the Brucella genus, common clinically encountered mycobacterial species, as well as common Salmonella serotypes (Gopaul et al., 2008; Dalmasso et al., 2009, 2010; Ben-Darif et al., 2010; Wang et al., 2010). Primer extension reaction assays have also been developed for typing of Listeria monocytogenes and Escherichia coli O157 (Van Stelten et al., 2010; Haugum et al., 2011; Lomonaco et al., 2011) as well as for rapid identification or differentiation of Lactobacillus casei and Lactobacillus plantarum (Huang et al., 2011a,b). Therefore, the purpose of the present work was to design and develop a multiplex minisequencing assay able to rapidly differentiate different groups of B. animalis ssp. lactis strains in pure culture and when present as a single strain in yogurt products.

\section{MATERIALS AND METHODS}

\section{Strains and DNA Extraction}

To test the ability of the method to correctly differentiate B. animalis ssp. lactis (BAL), 25 BAL strains representing 9 distinct genotypic groups were selected for use in this study (Table 1). All strains were grown for $18 \mathrm{~h}$ in $10 \mathrm{~mL}$ of de Man, Rogosa, and Sharpe (de Man et al., 1960) supplemented with $0.05 \%$ L-cysteine hydrochloride (MRSC broth). Cells were harvested and genomic DNA was extracted using the Wizard Genomic DNA Purification Kit (Promega, Madison, WI), according to the manufacturer's instructions
The ability of this method to distinguish BAL from other species of Bifidobacterium commonly added to probiotic-containing dairy products was evaluated by analysis of frozen cultures of Bifidobacterium longum ssp. infantis (BB-02) and Bifidobacterium longum ssp. longum (BB-46; Chr. Hansen, Hørsholm, Denmark). DNA was extracted from the pure cultures of each organism alone, when combined together (1:1), and when mixed with $B$. animalis ssp. lactis BB12 (1:1:1) using the PowerFood Microbial DNA Isolation Kit (MoBio, Carlsbad, CA). Following isolation, the DNA was subjected to multiplex preliminary PCR and minisequencing. In both cases, DNA was quantified by spectrophotometry (Biophotometer 6131, Eppendorf AG, Hamburg, Germany) and stored at $-20^{\circ} \mathrm{C}$ before use.

\section{Primer Design}

Six loci containing SNP able to discriminate between different BAL groups, as described in Briczinski et al. (2009), were selected as targets for this study. Sequences of internal fragments in these loci were aligned using Mega 5.0 software (Tamura et al., 2007), and 6 SNP that were deemed able to differentiate among $B$. animalis ssp. lactis were selected for further testing (Table 2). Primers for the preliminary multiplex PCR were designed approximately 200 to 300 nucleotides upstream and downstream from the diagnostic SNP (Table 2). Additionally, primers were designed so that the amplification products had different sizes to allow visualization following multiplex PCR.

For the multiplex PER, extension primers (EP) were designed immediately adjacent, upstream or downstream, to the selected SNP (Table 2). To allow efficient separation during capillary electrophoresis, 2 of the $6 \mathrm{EP}$ were designed with poly (dT) tails attached to their $5^{\prime}$ ends (Table 2). All EP were evaluated individually before testing in multiplex. Primers were synthesized by the Penn State Genomics Core Facility and Integrated DNA Technologies (Coralville, IA).

\section{Preliminary and Multiplex PCR}

Specificities of primers targeting the loci of interest were assessed by simplex PCR using DNA from DSM 10140 and Bl-04. Amplifications were performed in a final volume of $50 \mu \mathrm{L}$ containing $75 \mathrm{~m} M$ Tris- $\mathrm{HCl}(\mathrm{pH}$ 8.8), 1 unit of recombinant Taq DNA Polymerase (Invitrogen, Carlsbad, CA), $0.2 \mathrm{~m} M$ each of dATP, dCTP, dGTP, and dTTP (Pharmacia, Uppsala, Sweden), $5 \mu M$ of each primer, $1.5 \mathrm{mM} \mathrm{MgCl}_{2}$, and $0.5 \mathrm{mM}(250 \mathrm{ng})$ of DNA template. Once primer specificity was verified, multiplex PCR was performed on DNA extracted from all strains of interest to detect potentially undesirable 
Table 1. Description of the 25 Bifidobacterium animalis ssp. lactis strains analyzed in this study and the resulting 8 SNP profiles

\begin{tabular}{|c|c|c|c|}
\hline Strain $^{1}$ & $\begin{array}{c}\text { Corrected } \\
\text { Briczinski et al. } \\
\text { (2009) group }\end{array}$ & $\begin{array}{l}\text { Minisequencing } \\
\text { profile }\end{array}$ & $\begin{array}{l}\text { SNP profile no. } \\
\text { (this work) }\end{array}$ \\
\hline DSM 10140 & 1 & GATTTG & BAL_SNP_1 \\
\hline RB 1280 & 2 & GGTTTG & BAL_SNP_2 \\
\hline RB 1573 & 3 & AGTTTG & BAL_SNP_3 \\
\hline RB 4052 & 3 & AGTTTG & BAL_SNP_3 \\
\hline RB 4536 & 3 & AGTTTG & BAL_SNP_3 \\
\hline RB 7339 & 3 & AGTTTG & BAL_SNP_3 \\
\hline RB 9321 & 3 & AGTTTG & BAL_SNP_3 \\
\hline BB-12 & 3 & AGTTTG & BAL_SNP_3 \\
\hline RB 5851 & $3^{2}$ & AGTTTG & BAL_SNP_3 \\
\hline RB 4753 & $3^{2}$ & AGTTTG & BAL_SNP_3 \\
\hline RB 1791 & $4^{2}$ & AGTCTG & BAL_SNP_4 \\
\hline RB 7239 & 4 & AGTCTG & BAL_SNP_4 \\
\hline HN019 & 8 & AATTTG & BAL_SNP_5 \\
\hline RB 4825 & 9 & AGGTCA & BAL_SNP_6 \\
\hline RB 5251 & 9 & AGGTCA & BAL_SNP_6 \\
\hline Bl-04 & 9 & AGGTCA & BAL_SNP_6 \\
\hline RB 5859 & $9^{2}$ & AGGTCA & BAL_SNP_6 \\
\hline RB 3046 & $9^{2}$ & AGGTCA & BAL_SNP_6 \\
\hline RB 5422 & $9^{2}$ & AGGTCA & BAL_SNP_6 \\
\hline RB 1281 & 12 & AGTTCA & BAL_SNP_7 \\
\hline RB 5733 & 12 & AGTTCA & BAL_SNP_7 \\
\hline RB 8613 & 12 & AGTTCA & BAL_SNP_7 \\
\hline RB 9632 & 12 & AGTTCA & BAL_SNP_7 \\
\hline RB 0171 & 13 & AGTTCA & BAL_SNP_7 \\
\hline ATCC 27536 & 14 & AGTTCG & BAL_SNP_8 \\
\hline
\end{tabular}

${ }^{1} \mathrm{RB}$ strains were obtained from the strain collection at Penn State University (University Park); DSM 10140 was obtained from The German Collection of Microorganisms and Cell Cultures (Braunschweig, Germany); ATCC 27536 was obtained from the American Type Culture Collection (Manassas, VA); BB-12 was obtained from Chr. Hansen (Milwaukee, WI); HN019 and Bl-04 were obtained from DuPont Nutritional and Health (Madison, WI)

${ }^{2}$ During this study, the corresponding strains did not yield the expected SNP profile reported by Briczinski et al. (2009). Resequencing of the DNA from that study revealed data transposition errors. The correct groupings are given in the second column of this table.

pairings of primers. Multiplex PCR reactions were carried out as described above with $5 \mu M$ concentrations of each primer except for INDEL2_F/R, which was used at $10 \mu M$. Amplifications were performed using a Mastercycler pro thermocycler (Eppendorf North America Inc., Hauppauge, NY) beginning with 5 min at $95^{\circ} \mathrm{C}$ followed by 30 cycles at $95^{\circ} \mathrm{C}$ for $30 \mathrm{~s}, 58^{\circ} \mathrm{C}$ for $1 \mathrm{~min}, 72^{\circ} \mathrm{C}$ for $2 \mathrm{~min}$, and a final extension of $72^{\circ} \mathrm{C}$ for $7 \mathrm{~min}$. Amplicons were resolved by electrophoresis on a $2.0 \%$ agarose gel (Invitrogen) and visualized on a UV transilluminator and images captured by an AlphaImager 3300 gel documentation system (Alpha Innotech, San Leandro, CA).

\section{Primer Extension Reaction}

Multiplex PCR products were used as templates for subsequent PER following enzymatic clean up to remove unincorporated deoxynucleotide triphosphates (dNTP) and primers. To clean up the samples, $2 \mu \mathrm{L}$ of Exo-Sap (Affymetrix, Santa Clara, CA) was added to $5 \mu \mathrm{L}$ of PCR products, incubated at $37^{\circ} \mathrm{C}$ for $1 \mathrm{~h}$, and then heated to $80^{\circ} \mathrm{C}$ for $15 \mathrm{~min}$ to inactive residual enzyme.

The EP were first tested individually to evaluate performance and validate their molecular size. Simplex PER were performed in a total volume of $10 \mu \mathrm{L}$ according to the SNaPshot multiplex kit protocol (Applied Biosystems, Foster City, CA) with the following minor modifications: $3 \mu \mathrm{L}$ of purified preliminary PCR products, $3 \mu \mathrm{L}$ of SnaPshot Multiplex Ready Reaction Mix, and EP at a final concentration of $0.2 \mu M$.

Subsequently, multiplex PER were performed using $0.1 \mu M$ each of sequencing primers EP1, EP3, EP4, and EP6 and $0.6 \mu M$ of sequencing primers EP2 and EP5. The concentration of each primer was chosen based on results from preliminary assays. The minisequencing reactions consisted of 25 cycles with the following conditions: denaturation at $96^{\circ} \mathrm{C}$ for $10 \mathrm{~s}$, annealing at $50^{\circ} \mathrm{C}$ for $5 \mathrm{~s}$, and extension at $60^{\circ} \mathrm{C}$ for $30 \mathrm{~s}$. Reactions were performed using a Mastercycler pro thermocycler (Eppendorf North America Inc.). 
Table 2. Polymerase chain reaction and extension primers (EP), fragment sizes, and allelic locations for the 6 Bifidobacterium animalis ssp. lactis genomic regions and the 6 SNP interrogated in this study

\begin{tabular}{|c|c|c|c|c|}
\hline Reaction & Primer & Primer sequence $\left(5^{\prime}-3^{\prime}\right)$ & $\begin{array}{l}\text { Fragment } \\
\text { size (bp) }\end{array}$ & $\begin{array}{c}\text { Allelic location on } \\
\text { strain DSM 10140 } \\
\text { (GenBank: CP001606) }\end{array}$ \\
\hline \multirow[t]{12}{*}{${\text { Preliminary } \mathrm{PCR}^{1}}$} & Balat_0051F & 5'-GAGACCGTCATCGAGTCC-3' & 783 & $53,234-53,723$ \\
\hline & Balat_0051R & 5'-CACTGAGCGAGTAATCACAGGA-3' & & \\
\hline & $\operatorname{igr} 6 \mathrm{~F}$ & 5'-TGCGAGATGAAACGGATATG-3' & 368 & $686,118-687,770$ \\
\hline & igr6R & 5'-ACCTTCGTATGGTCGAGCTG-3' & & \\
\hline & Balat_0710F & 5'-GCATTGGTTCAGGGTCTGTT-3' & 418 & $839,211-839,628$ \\
\hline & Balat_0710R & 5'-GTAGCCCCAATGGTCGAAT-3' & & \\
\hline & INDEL2_F & 5'-AACCGTCTGCTGCTGTTTCT-3' & $511 / 457$ & $902,690-903,183$ \\
\hline & INDEL2_R & 5'-GGTTGGCTTTCTGCCAAT-3' & & \\
\hline & $g l c U \_\mathrm{F}$ & 5'-GTCCGGCGCATTGTAGATAG-3' & 627 & $1,259,721-1,260,328$ \\
\hline & $g l c U \_\mathrm{R}$ & 5'-CGTACAACGATTTGGCATTG-3' & & \\
\hline & igr9_F & 5'-GTTTCGAGGCCTGGTATTGA-3' & 730 & $1,636,223-1,636,935$ \\
\hline & igr9_R & 5'-ATCACGTGGTTGCCTTGC-3' & & \\
\hline \multirow{6}{*}{ Primer extension reaction (PER) } & EP1 (Balat_0051) & $5^{\prime}$-CGGCGCCCACCGCCTCA-3' & $17+1$ & $53,304-53,320$ \\
\hline & EP2 (igr9) & 5'-GTTGCCGTTCTGCTTCAG-3' & $18+1$ & $1,636,587-1,636,604$ \\
\hline & EP3 $\left(\right.$ INDEL2) ${ }^{2}$ & 5'-TGTGTTCCGACATCATCTTCGACC-3' & $24+1$ & $902,969-902,946$ \\
\hline & EP4 (glcU) & 5'-ACAACGCAATGAGCATGCCCATGTGAAG-3' & $28+1$ & $1,260,287-1,260,314$ \\
\hline & EP5 (Balat_0710) & 5'-(18T)CGCGCTCGTCGCCGGCGTGG-3' & $38+1$ & $839,408-839,427$ \\
\hline & EP6 (igr6) & 5'-(33T)CTAGTAAAGATGTGCGGGCA-3' & $53+1$ & $686,979-686,998$ \\
\hline
\end{tabular}

These regions represented intergenic regions 6 and 9 (igr6 and igr9), a transposase (Balat_0051), a hypothetical membrane protein (Balat_0710), a putative glucose uptake permease $(g l c U)$, and an insertion/deletion in the long-chain fatty acid-CoA ligase (INDEL2).

${ }^{2}$ Primer was designed in a reverse position. 
The final volume of the PER $(10 \mu \mathrm{L})$ was treated post-extension with 1 unit of calf intestinal alkaline phosphatase (CIAP; Fermentas, Burlington, CA) and incubated at $37^{\circ} \mathrm{C}$ for $1 \mathrm{~h}$ followed by $15 \mathrm{~min}$ at $75^{\circ} \mathrm{C}$. This was carried out to remove the $5^{\prime}$-phosphoryl group of the $[\mathrm{F}] \mathrm{ddNTP}$ (where $\mathrm{F}=$ fluorescent) of unincorporated $[\mathrm{F}] \mathrm{ddNTP}$ that could otherwise co-migrate with the extended primers and produce a high background signal.

Finally, $1 \mu \mathrm{L}$ of the post-extension purified PER product was mixed with $24.6 \mu \mathrm{L}$ of $\mathrm{Hi}$-Di formamide and $0.4 \mu \mathrm{L}$ of GeneScan 120 LIZ size standard (Applied Biosystems). Each multiplex minisequencing reaction was analyzed using an ABI 310 Genetic Analyzer (Applied Biosystems), at the Penn State Genomics Core Facility (University Park, PA). Electropherograms were evaluated with PeakScanner 1.0 software (Applied Biosystems), available at http://www.lifetechnologies. com/order/catalog/product/4381867.

The discriminatory power (D) of the method (Hunter and Gaston, 1988) with 95\% confidence intervals was calculated by EpiCompare version 1.0 (Ridom, Münster, Germany).

\section{Product Production and Analysis}

To test the applicability of the method for the analysis of probiotic-supplemented dairy products, total genomic DNA was directly extracted from yogurt samples. A yogurt-based strawberry smoothie was manufactured with and without the addition of $B$. animalis ssp. lactis BB12 at a level of $\sim 1.30 \times 10^{8} \mathrm{cfu} / \mathrm{g}$. These products were manufactured as part of a previous (Merenstein et al., 2011) and an ongoing study; compositional details are outlined in Table 3. Viable counts of B. animalis ssp. lactis were determined by pour-plating on MRS-NNPL (nalidixic acid, neomycin sulfate, lithium chloride, and paramomycin sulfate; Laroia and Martin, 1991) with growth under anaerobic conditions at $37^{\circ} \mathrm{C}$ for $72 \mathrm{~h}$. Total DNA was extracted from both products using the PowerFoods DNA extraction kit (MoBio) according to the manufacturer's instructions. Subsequently, isolated DNA was subjected to minisequencing analysis, as detailed above. In addition, DNA extracted from each product was evaluated using the subspecies-specific primers Bflact2/5 (Ventura et al., 2001) and genusspecific primers Lm3/26 (Ventura and Zink, 2002).

\section{RESULTS}

\section{Primer Design and PCR}

Analysis of the alignment of the BAL sequences obtained from Briczinski et al. (2009) confirmed that
Table 3. Product composition of strawberry yogurt drink evaluated for Bifidobacterium animalis ssp. lactis content

\begin{tabular}{lc}
\hline Component & $\begin{array}{c}\text { Composition in } \\
\text { yogurt drink }(\%)\end{array}$ \\
\hline Fat & 1.0 \\
Milk solids nonfat & 6.8 \\
Sucrose & 6.1 \\
Corn syrup solids & 6.4 \\
Pectin & 0.4 \\
Strawberry puree & 3.0 \\
TS & 23.7 \\
\hline
\end{tabular}

the combination of selected SNP could provide profiles specific for 8 BAL groups (Table 1). Primers tested in simplex reactions produced amplicons of the expected size (Figure 1). All evaluated strains yielded amplicons of the expected size (368 bp for igr6, $730 \mathrm{bp}$ for igr9, 490 bp for Balat_0051, 418 bp for Balat_0710, 627 bp for $g l c U$, and $511 \mathrm{bp}$ for INDEL2). Amplicons from reactions with DSM 10140 and Bl-04 are shown in Figure 1.

After verification in simplex reactions, the primers were combined and used in multiplex PCR. Evaluation of the PCR amplicons from multiplex reactions using DSM 10140 and Bl-04 are shown in lanes 1 to 6 and lanes 9 to 14 of Figure 1, respectively. Analysis of non-BAL species BB-02 and BB- 46 revealed 3 and 2 amplicons, respectively (data not shown).

\section{Minisequencing}

For all BAL strains, simplex minisequencing yielded a peak of the expected color - green (adenine, A), black (cytosine, C), blue (guanine, G) and red (thymine, T) - specific to the fluorescently labeled [F]ddNTP incorporated at the diagnostic SNP site. Following optimization, multiplex PER were performed on DNA extracted from all BAL strains, yielding 8 specific patterns (Figure 2, Table 1). Representative chromatograms for each of the 8 distinct BAL_SNP groups are shown in Figure 2. The SNP-specific extension primers were designed for simultaneous annealing and single nucleotide extension, and their length ranged from 17 to $39 \mathrm{bp}$ to allow simultaneous resolution via capillary electrophoresis. Following optimization, the preliminary multiplex PCR products served as template for subsequent multiplex PER reactions, performed using the mixture of the 6 extension primers. All evaluated strains gave the expected profiles, giving rise to 8 specific patterns (Figure 2, Table 1). With the exception of BAL_SNP_7, the PER data corresponded to one SNP profile of the corrected Briczinski et al. (2009) typing scheme (Table 1). 


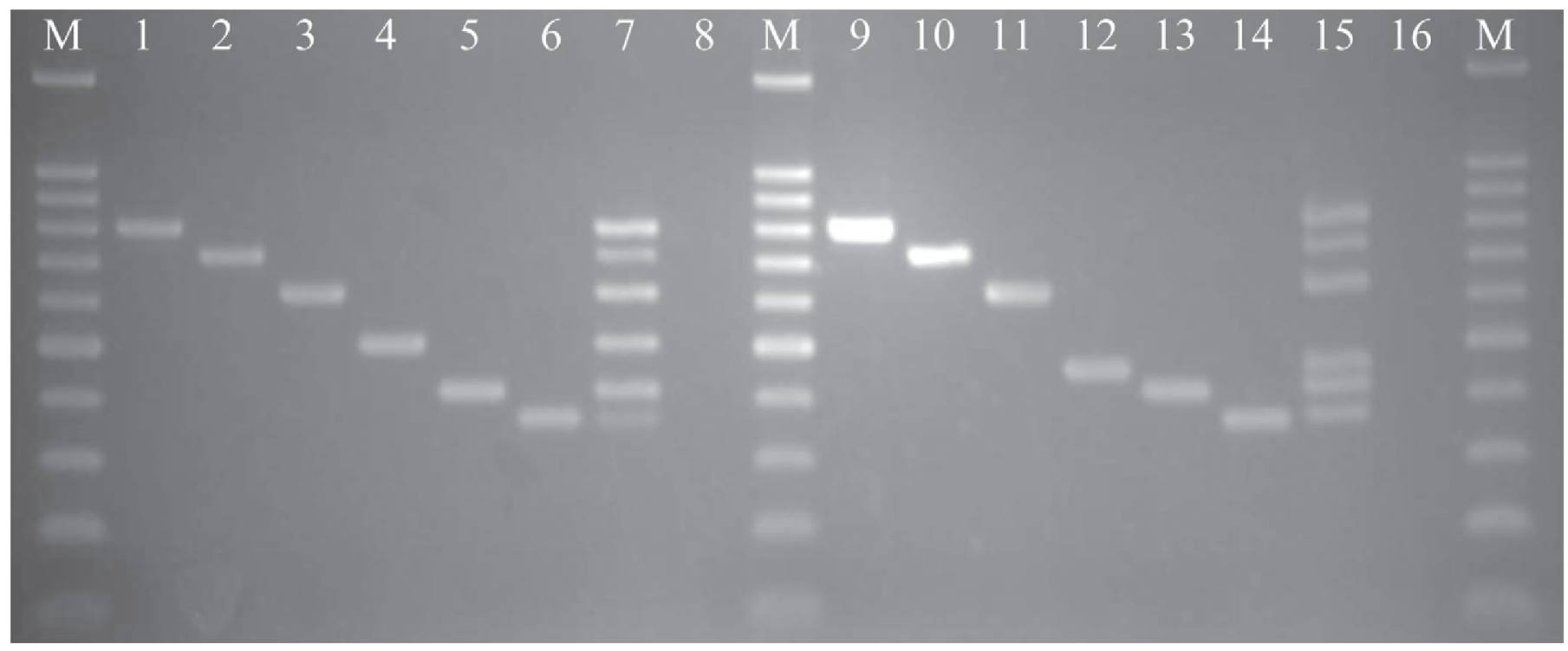

Figure 1. Preliminary multiplex amplification of the 6 Bifidobacterium animalis ssp. lactis genomic regions: igr6, igr9, Balat_0051, Balat_0710, $g l c U$, and INDEL2 in strains DSM 10140 and Bl-04. M = 100-bp DNA ladder; lane 1 (primer/locus) = DSM 10140/Balat_0051; lane $2=$ DSM 10140/igr9; lane 3 = DSM 1014/glcU; lane $4=$ DSM 10140/INDEL2; lane $5=$ DSM 10140/Balat_0710; lane $6=$ DSM 10140/igr6; lane $7=$ multiplex; lane 8 = negative control; lane 9 = Bl-04/ Balat_0051; lane 10 = Bl-04/igr9; lane $11=$ Bl-04/glcU; lane 12 = Bl-04/INDEL2; lane 13 = Bl-04/Balat_0710; lane $14=$ Bl-04/igr6; lane $15=$ multiplex; lane $16=$ negative control.

Minisequencing for the 2 non-BAL species, BB-02 and BB-46, did not yield any peaks, regardless of whether they were pure or in a mixture. When BB-02 and BB46 were combined with BAL strain BB-12, the expected chromatogram was obtained (data not shown).

\section{Differentiation of Strains}

Using one isolate for each of the profiles listed in Table 1, a discriminatory power of 0.97 was calculated with a $95 \%$ confidence interval of 0.74 to 0.90 . This indicates that the method provided a $97 \%$ chance that analysis of any 2 randomly selected strains of this closely related strain collection would be placed into 2 different groups.

\section{Utilization of the Method to Assess a Yogurt Product}

The minisequencing protocol was applied to drinkable strawberry yogurt products manufactured with and without $B$. animalis ssp. lactis BB-12. Viable bifidobacteria cells counted on MRS-NNLP agar were present at a level of $1.65 \times 10^{8} \mathrm{cfu} / \mathrm{mL}$ in the yogurt drink manufactured with addition of BB-12 and were undetectable in the yogurt drink manufactured without BB-12. Analysis of total DNA extracted from yogurt drink samples containing BB-12 by PCR using genusand subspecies-specific primers resulted in amplicons of appropriate size, indicating the presence of DNA from the organism. Analysis of DNA from yogurt manufactured without BB-12 did not result in amplification (data not shown). Minisequencing applied to DNA from yogurt manufactured with BB-12 resulted in the profile of AGTTTG, appropriately corresponding to BAL_SNP_3, which contains B. animalis ssp. lactis BB-12 (see Table 2).

\section{DISCUSSION}

Detection of minisequencing products may require as little as 18 min compared with the $2.5 \mathrm{~h}$ required for capillary electrophoresis of sequencing products. Minisequencing also yields results that are simple to analyze and interpret. Additionally, by developing a multiplex preliminary PCR assay to simultaneously amplify all the loci of interest, the time and expense required to complete the analysis would be reduced. Identification and differentiation of specific strains of $B$. animalis ssp. lactis has traditionally been difficult and unreliable due to the high degree of relatedness between strains of interest. Pulsed-field gel electrophoresis has been considered the gold standard for the differentiation of bacterial strains (FAO/WHO, 2002); however, PFGE does little to resolve strains of this monomorphic subspecies (Mayer et al., 2007; Briczinski et al., 2009). In this work, a SNP-INDEL typing scheme developed in our laboratory (Briczinski et al., 2009) was adapted for minisequencing analysis. Notably, minisequencing 

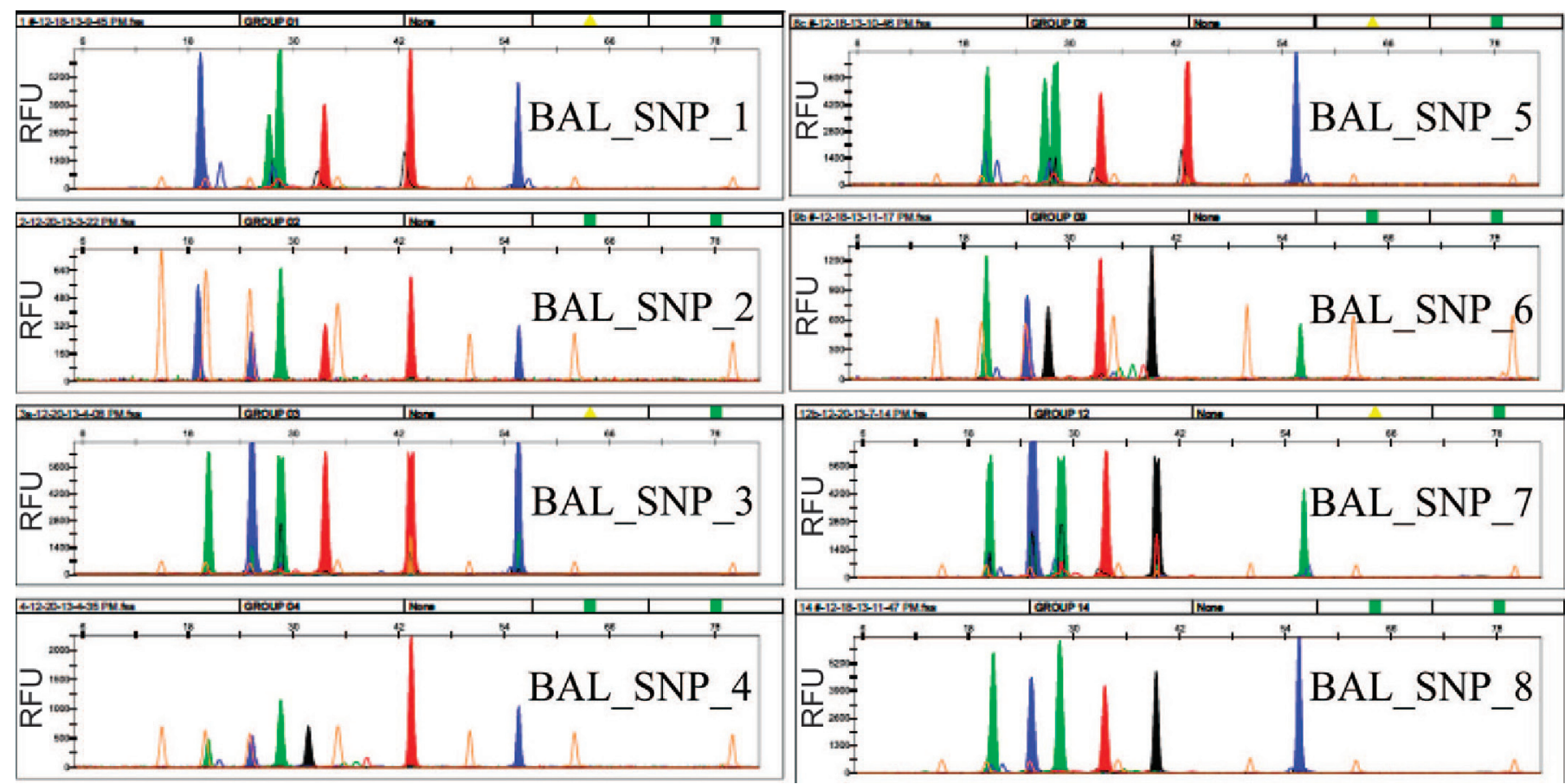

Figure 2. Representative chromatograms for each of the specific profiles obtained by multiplex primer extension reaction (PER) of strains of Bifidobacterium animalis ssp. lactis. Minisequencing peak colors are green (adenine, A), black (cytosine, C), blue (guanine, G), and red (thymine, $\mathrm{T}) . \mathrm{RFU}=$ relative fluorescence units (unitless).

can only be used to discriminate those species or strains for which DNA sequence data are already available. The incorporation of new SNP in existing SNP-typing schemes would likely be required to differentiate newly defined strains or groups of strains. Such SNP would have to be identified relying on traditional sequencing data. Over the last few years, minisequencing approaches have been successfully developed for identification or characterization of bacterial species belonging to a variety of different genera (Gopaul et al., 2008; Dalmasso et al., 2009; Ben-Darif et al., 2010; Wang et al., 2010; Lomonaco et al., 2011).

Previous work with $B$. animalis ssp. lactis identified 14 distinct genomic clusters among a collection of 24 strains of $B$. animalis ssp. lactis (Briczinski et al., 2009). However, the current analysis resolved the same collection of strains into 8 genomic clusters. In particular, during the current study, some strains (Table 1) did not give rise to the expected SNP profile reported by Briczinski et al. (2009). Resequencing of the DNA from that study revealed sequence data transposition errors. Reanalysis of the corrected sequence data revealed that 9 groups were observed by Briczinski et al. (2009).

Discriminatory power expresses the probability that 2 unrelated strains randomly sampled in the analyzed population will be assigned a different type by the typing system. Therefore, only 1 isolate for each profile was used for the calculation. The assay developed herein was able to discriminate 8 out of 9 previously identified distinct genomic clusters (Briczinski et al., 2009) and therefore yielded a discriminatory power of 0.97. The PER method developed here was not able to resolve groups 12 and 13, which were originally separated based on the different number of $G$ residues ( 7 or 8) at the locus Balat_0141 (Briczinski et al., 2009). However, the sequence surrounding this SNP in groups 12 and 13 in both the forward and reverse directions was identical, and thus these 2 groups were combined into the BAL_SNP_7 group in this work. Discrimination between strains belonging to this group can be achieved by Sanger sequencing of the discriminating locus that cannot be resolved by our developed minisequencing assay. Regarding the BAL_SNP_3 group, whole-genome sequencing could be used to achieve deeper discrimination. However, it may not be possible to further discriminate the strains within this group as they could represent different isolates of the same strain, as is known for the chronologically distinct isolates RB 4825 and Bl-04 (Briczinski et al., 2009).

Direct analysis of a drinkable strawberry yogurt demonstrated the utility of this method to detect a single strain of B. animalis ssp. lactis present in a yogurt smoothie. The product made without bifidobacteria showed no amplification using the 6 preliminary PCR 
primers, highlighting the lack of cross-reactivity with traditional yogurt cultures. This was further confirmed by the lack of a positive reaction with genus- and subspecies-specific primers. The lack of cross-reactivity with cultures of $B$. longum ssp. longum and B. longum ssp. infantis was also confirmed, as the preliminary PCR did not show amplification for all targeted genes and therefore that sample would not be subjected to the minisequencing assay (which would result in no peaks, in any case). This method could be used to identify a culture supplier's own strain in a customer's products. This method may also be used to lend credence to clinical trials, when $B$. animalis ssp. lactis is being used as an intervention. The assay developed herein can be used to prove that a recovered strain is the intervention strain and not a strain already present in the panelist (i.e., a participant in a clinical study consuming the strain of interest).

\section{CONCLUSIONS}

Traditionally, identification of $B$. animalis ssp. lactis strains has been difficult and time consuming. In the method described here, we were able to directly purify all 6 PCR products from a single multiplex PCR reaction before minisequencing, resulting in savings of reagents and time. Additionally, we demonstrated the value of this method in the identification of BAL_SNP groups directly from a yogurt product without plating on expensive selective media. The method described here can be used in research laboratories, clinical trials evaluating the efficacy of $B$. animalis ssp. lactis, or in commercial products to identify commercial strains utilized in the industry.

\section{ACKNOWLEDGMENTS}

The authors thank the Penn State University Genomics Core Facility for assistance with sequencing and SNaPShot analysis, and thank Edward Dudley (Department of Food Science, The Pennsylvania State University, University Park) for his helpful and insightful comments. The authors also thank Zhaoyong Ba Department of Food Science, The Pennsylvania State University, University Park) for assistance with yogurt manufacture.

\section{REFERENCES}

Barrangou, R., E. P. Briczinski, L. L. Traeger, J. R. Loquasto, M. Richards, P. Horvath, A. C. Coute-Monvoisin, G. Leyer, S. Rendulic, J. L. Steele, J. R. Broadbent, T. Oberg, E. G. Dudley, S. Schuster, D. A. Romero, and R. F. Roberts. 2009. Comparison of the complete genome sequences of Bifidobacterium animalis ssp. lactis DSM 10140 and Bl-04. J. Bacteriol. 191:4144-4151. http:// dx.doi.org/10.1128/JB.00155-09.

Ben-Darif, E., F. Jury, E. De Pinna, E. J. Threlfall, F. J. Bolton, A. J. Fox, and M. Upton. 2010. Development of a multiplex primer extension assay for rapid detection of Salmonella isolates of diverse serotypes. J. Clin. Microbiol. 48:1055-1060. http://dx.doi. org/10.1128/JCM.01566-09.

Bottacini, F., F. Dal Bello, F. Turroni, C. Milani, S. Duranti, E. Foroni, A. Viappiani, F. Strati, D. Mora, D. van Sinderen, and M. Ventura. 2011. Complete genome sequence of Bifidobacterium animalis ssp. lactis BLC1. J. Bacteriol. 193:6387-6388. http:// dx.doi.org/10.1128/JB.06079-11.

Briczinski, E. P., J. R. Loquasto, R. Barrangou, E. G. Dudley, A. M. Roberts, and R. F. Roberts. 2009. Strain-specific genotyping of Bifidobacterium animalis ssp. lactis by using single-nucleotide polymorphisms, insertions, and deletions. Appl. Environ. Microbiol. 75:7501-7508. http://dx.doi.org/10.1128/AEM.01430-09.

Briczinski, E. P., and R. F. Roberts. 2006. Technical note: A rapid pulsed-field gel electrophoresis method for analysis of Bifidobacteria. J. Dairy Sci. 89:2424-2427.

Chervaux, C., C. Grimaldi, A. Bolotin, B. Quinquis, S. Legrain-Raspaud, J. E. van Hylckama Vlieg, G. Denariaz, and T. Smokvina. 2011. Genome sequence of the probiotic strain Bifidobacterium animalis ssp. lactis CNCM I-2494. J. Bacteriol. 193:5560-5561. http://dx.doi.org/10.1128/JB.05716-11.

Dalmasso, A., T. Civera, and M. T. Bottero. 2009. Multiplex primerextension assay for identification of six pathogenic vibrios. Int. J. Food Microbiol. 129:21-25. http://dx.doi.org/10.1016/j.ijfoodmicro.2008.10.029

Dalmasso, A., K. Rantsiou, L. Cocolin, and M. T. Bottero. 2010. Development of a biomolecular assay for the identification of Listeria at species level. Foodborne Pathog. Dis. 7:565-571. http:// dx.doi.org/10.1089/fpd.2009.0456.

de Man, J. D., M. Rogosa, and M. E. Sharpe. 1960. A medium for the cultivation of lactobacilli. J. Appl. Bacteriol. 23:130-135.

FAO/WHO. 2002. Guidelines for the evaluation of probiotics in food. Report of a joint FAO/WHO working group on drafting guidelines for the evaluation of probiotics in food. London, ON, Canada, 2002. Accessed Sep. 19, 2014. ftp://ftp.fao.org/es/esn/food/ wgreport2.pdf.

Garrigues, C., E. Johansen, and M. B. Pedersen. 2010. Complete genome sequence of Bifidobacterium animalis ssp. lactis BB-12, a widely consumed probiotic strain. J. Bacteriol. 192:2467-2468. http://dx.doi.org/10.1128/JB.00109-10.

Gopaul, K. K., M. S. Koylass, C. J. Smith, and A. M. Whatmore. 2008. Rapid identification of Brucella isolates to the species level by real time PCR based single nucleotide polymorphism (SNP) analysis. BMC Microbiol. 8:86. http://dx.doi.org/10.1186/14712180-8-86.

Haugum, K., L. T. Brandal, I. Lobersli, G. Kapperud, and B. A. Lindstedt. 2011. Detection of virulent Escherichia coli O157 strains using multiplex PCR and single base sequencing for SNP characterization. J. Appl. Microbiol. 110:1592-1600. http://dx.doi. org $/ 10.1111 / \mathrm{j} .1365-2672.2011 .05015 . x$.

Huang, C. H., M. T. Chang, M. C. Huang, and F. L. Lee. 2011a. Application of the SNaPshot minisequencing assay to species identification in the Lactobacillus casei group. Mol. Cell. Probes 25:153-157. http://dx.doi.org/10.1016/j.mcp.2011.03.002.

Huang, C. H., M. T. Chang, M. C. Huang, and F. L. Lee. 2011b. Rapid identification of Lactobacillus plantarum group using the SNaPshot minisequencing assay. Syst. Appl. Microbiol. 34:586-589. http://dx.doi.org/10.1016/j.syapm.2011.02.006.

Hunter, P. R., and M. A. Gaston. 1988. Numerical index of the discriminatory ability of typying systems: an application of Simpson's index of diversity. J. Clin. Microbiol. 26:2465-2466.

Jian, W., L. Zhu, and X. Dong. 2001. New approach to phylogenetic analysis of the genus Bifidobacterium based on partial HSP60 gene sequence. Int. J. Syst. Evol. Microbiol. 51:1633-1638.

Kim, J. F., H. Jeong, D. S. Yu, S. H. Choi, C. G. Hur, M. S. Park, S. H. Yoon, D. W. Kim, G. E. Ji, H. S. Park, and T. K. Oh. 2009 Genome sequence of the probiotic bacterium Bifidobacterium ani- 
malis ssp. lactis AD011. J. Bacteriol. 191:678-679. http://dx.doi. org/10.1128/JB.01515-08.

Laroia, S., and J. H. Martin. 1991. Methods for enumerating and propagating bifidobacteria. Cult. Dairy Prod. J. 26:32-34.

Lomonaco, S., S. J. Knabel, A. Dalmasso, T. Civera, and M. T. Bottero. 2011. Novel multiplex single nucleotide polymorphism-based method for identifying epidemic clones of Listeria monocytogenes. Appl. Environ. Microbiol. 77:6290-6294. http://dx.doi. org/10.1128/AEM.00429-11.

Loquasto, J. R., R. Barrangou, E. G. Dudley, B. Stahl, C. Chen, and R. F. Roberts. 2013. Bifidobacterium animalis ssp. lactis ATCC 27673 is a genomically unique strain within this conserved subspecies. Appl. Environ. Microbiol. 79:6903-6910. http://dx.doi. org/10.1128/AEM.01777-13.

Marteau, P., E. Cuillerier, S. Meanace, M. F. Gerhardt, A. Myara, M. Bouvier, C. Bouley, F. Tondu, G. Bommelaer, and J. C. Grimaud. 2002. Bifidobacterium animalis strain DN-173 010 shortens the colonic transit time in healthy women: A double-blind, randomized, controlled study. Aliment. Pharmacol. Ther. 16:587-593.

Masco, L., M. Ventura, R. Zink, G. Huys, and J. Swings. 2004. Polyphasic taxonomic analysis of Bifidobacterium animalis and Bifidobacterium lactis reveals relatedness at the subspecies level: Reclassification of Bifidobacterium animalis as Bifidobacterium animalis ssp. animalis ssp. nov. and Bifidobacterium lactis as Bifidobacterium animalis ssp. lactis ssp. nov. Int. J. Syst. Evol. Microbiol. $54: 1137-1143$

Matsumoto, M., H. Ohishi, and Y. Benno. 2004. $\mathrm{H}^{+}$-ATPase activity in Bifidobacterium with special reference to acid tolerance. Int. J. Food Microbiol. 93:109-113.

Mayer, H. K., E. Amtmann, E. Philippi, G. Steinegger, S. Mayrhofer, and W. Kneifel. 2007. Molecular discrimination of new isolates of Bifidobacterium animalis ssp. lactis from reference strains and commercial probiotic strains. Int. Dairy J. 17:565-573. http:// dx.doi.org/10.1016/j.idairyj.2006.05.008.

Meile, L., W. Ludwig, U. Reuger, C. Gut, P. Kaufmann, G. Dasen, S. Wenger, and M. Teuber. 1997. Bifidobacterium lactis sp. nov., a moderately oxygen tolerant species isolated from fermented milk. Syst. Appl. Microbiol. 20:57-64. http://dx.doi.org/10.1016/ S0723-2020(97)80048-3.

Merenstein, D., J. Gonzalez, A. G. Young, R. F. Roberts, M. E. Sanders, and S. Petterson. 2011. Study to investigate the potential of probiotics in children attending school. Eur. J. Clin. Nutr. 65:447-453. http://dx.doi.org/10.1038/ejcn.2010.290.

Roy, D., and S. Sirois. 2000. Molecular differentiation of Bifidobacterium species with amplified ribosomal DNA restriction analysis and alignment of short regions of the $l d h$ gene. FEMS Microbiol. Lett. 191:17-24.

Simpson, P. J., C. Stanton, G. F. Fitzgerald, and R. P. Ross. 2005. Intrinsic tolerance of Bifidobacterium species to heat and oxygen and survival following spray drying and storage. J. Appl. Microbiol. 99:493-501.
Stahl, B., and R. Barrangou. 2012. Complete genome sequences of probiotic strains of Bifidobacterium animalis ssp. lactis B420 and Bi-07. J. Bacteriol. 194:4131-4132. http://dx.doi.org/10.1128/ JB.00766-12.

Sun, Z., X. Chen, J. Wang, P. Gao, Z. Zhou, Y. Ren, T. Sun, L. Wang, H. Meng, W. Chen, and H. Zhang. 2010. Complete genome sequence of probiotic Bifidobacterium animalis ssp. lactis strain V9. J. Bacteriol. 192:4080-4081. http://dx.doi.org/10.1128/JB.0036910.

Tamura, K., J. Dudley, M. Nei, and S. Kumar. 2007. MEGA4: Molecular Evolutionary Genetics Analysis (MEGA) Software Version 4.0. Mol. Biol. Evol. 24:1596-1599.

Van Stelten, A., J. M. Simpson, T. J. Ward, and K. K. Nightingale. 2010. Revelation by single-nucleotide polymorphism genotyping that mutations leading to a premature stop codon in inlA are common among Listeria monocytogenes isolates from ready-to-eat foods but not human listeriosis cases. Appl. Environ. Microbiol. 76:2783-2790. http://dx.doi.org/10.1128/AEM.02651-09.

Veiga, P., C. A. Gallini, C. Beal, M. Michaud, M. L. Delaney, A. DuBois, A. Khlebnikov, J. E. T. van Hylckama Vlieg, S. Punit, J. N. Glickman, A. Onderdonk, L. H. Glimcher, and W. S. Garrett. 2010. Bifidobacterium animalis ssp. lactis fermented milk product reduces inflammation by altering a niche for colitogenic microbes. Proc. Natl. Acad. Sci. USA 107:18132-18137. http://dx.doi org/10.1073/pnas.1011737107.

Ventura, M., R. Reniero, and R. Zink. 2001. Specific identification and targeted characterization of Bifidobacterium lactis from different environmental isolates by a combined multiplex-PCR approach. Appl. Environ. Microbiol. 67:2760-2765.

Ventura, M., and R. Zink. 2002. Rapid identification, differentiation, and proposed new taxonomic classification of Bifidobacterium lactis. Appl. Environ. Microbiol. 68:6429-6434.

Ventura, M., and R. Zink. 2003. Comparative sequence analysis of the tuf and recA genes and restriction fragment length polymorphism of the internal transcribed spacer region sequences supply additional tools for discriminating Bifidobacterium lactis from Bifidobacterium animalis. Appl. Environ. Microbiol. 69:7517-7522.

Vernazza, C. L., G. R. Gibson, and R. A. Rastall. 2006. Carbohydrate preference, acid tolerance and bile tolerance in five strains of Bifidobacterium. J. Appl. Microbiol. 100:846-853.

Waller, P. A., P. K. Gopal, G. J. Leyer, A. C. Ouwehand, C. Reifer, M. E. Stewart, and L. E. Miller. 2011. Dose-response effect of Bifidobacterium lactis HN019 on whole gut transit time and functional gastrointestinal symptoms in adults. Scand. J. Gastroenterol. 46:1057-1064. http://dx.doi.org/10.3109/00365521.2011.584895.

Wang, H., J. Yue, M. Han, J. Yang, and Y. Zhao. 2010. Rapid method for identification of six common species of mycobacteria based on multiplex SNP analysis. J. Clin. Microbiol. 48:247-250. http:// dx.doi.org/10.1128/JCM.01084-09. 